\title{
ENERGY OF ACTIVATION OF THE VT5 AND VT1-0 TITANIUM ALLOYS UNDER SHORT-TERM CREEP IN AIR AND ARGON
}

\author{
S. V. Smirnov, L. M. Zamaraev \\ Institute of Engineering Science, Ural Branch of the Russian Academy of Sciences, \\ 34 Komsomolskaya St., 620049, Ekaterinburg, Russia \\ Corresponding author. E-mail: leva.zam@mail.ru; \\ address for correspondence: ul. Komsomolskaya, 34, 620049, Ekaterinburg, Russian Federation. \\ Tel.: +7 (343) 37535 96; fax: +7 (343) 3745330
}

Test results for the short-term creep of commercially pure VT1-0 titanium and the VT5 alloy at temperatures ranging from $673 \mathrm{~K}$ to $1323 \mathrm{~K}$ and rated tensile stresses ranging between 4.45 and 9.36 $\mathrm{MPa}$ in air and argon are presented. The experimental results have shown a considerable decrease in the speed of creep under loading in the argon environment in comparison with that in air. The values of the activation energy determined in tests with a stepwise change of temperature by Dorn's method have close values for the materials studied in air and argon, 250 to $300 \mathrm{~kJ} / \mathrm{mol}$, and this testifies to the identical mechanism of creep.

Keywords: titanium alloys, environmental effect on creep, creep activation energy.

DOI: $10.17804 / 2410-9908.2016 .6 .100-110$

\section{References}

1. Kumar J., Raman S.G.S., Kumar V. Creep-Fatigue Interactions in Ti-6Al-4V Alloy at Ambient Temperature. Transactions of the Indian Institute of Metals, 2016, vol. 69, no. 2, pp. 349-352. DOI: $10.1007 / \mathrm{s} 12666-015-0766-7$.

2. Grabovetskaya G.P., Zabudchenko O.V., Stepanova E.N. Effect of hydrogen on the lowtemperature creep of a submicrocrystalline Ti-6Al-4V alloy. Russian Metallurgy (Metally), 2010, vol. 2010, no. 3, pp. 229-234. DOI: 10.1134/S0036029510030134.

3. $\quad \mathrm{Yu} \mathrm{H.,} \mathrm{Dong} \mathrm{C.,} \mathrm{Jiao} \mathrm{Z.,} \mathrm{Kong} \mathrm{F.,} \mathrm{Chen} \mathrm{Y.,} \mathrm{Su} \mathrm{Y.} \mathrm{High} \mathrm{temperature} \mathrm{creep} \mathrm{and} \mathrm{fatigue}$ behavior and life prediction method of a TiAl alloy. Acta Metallurgica Sinica, 2013, vol. 49, no. 11, pp. 1311-1317. DOI: 10.3724/SP.J.1037.2013.00434.

4. Rosen A., Rottem A. The effect of high temperature exposure on the creep resistance of Ti6Al4V alloy. Mater. Sci. Eng. A, 1976, vol. 22, iss. C, pp. 23-29. DOI: 10.1016/00255416(76)90132-4.

5. Aksenov Yu.A., Bashkin I.O., Kolmogorov V.L., Ponyatovskiy Ye.G., Taluts G.G., Kataya V.K., Levin I.V., Potapenko Yu.I., Trubin A.N. Influence of hydrogen on the plasticity and elastic stiffness of technical titanium VT1-0 at temperatures up to $750^{\circ} \mathrm{C}$. Physics of Metals and Metallography, 1989, vol. 67, no. 5, pp. 157-163.

6. $\quad$ Reis D.A.P., De Moura Neto C., Neto F.P., Barboza M.J.R., Da Silva C.R.M. The oxidation effect in the titanium alloy at high temperature. SAE Technical Papers, 2007.

7. Kolychev B.A. Vodorodnaya khrupkost metallov [Hydrogen Brittleness of Metals]. M., Metallurgiya Publ., 1985, 286 p. (In Russian).

8. Mekhanicheskie svoistva legkikh splavov pri temperaturakh $i$ skorostyakh obrabotki davleniem: spravochnik [Mechanical Properties of Light Alloys at Temperatures and Rates of Forming: reference book, P.G. Miklyaev, ed.]. M., Metallurgiya Publ., 1994, 280 p. (In Russian).

9. Alabort E., Kontis P., Barba D., Dragnevski K., Reed R.C. On the mechanisms of superplasticity in Ti-6Al-4V. Acta Materialia, 2016, vol. 105, no. 1, pp. 449-463. DOI: 10.1016/j.actamat.2015.12.003.

10. Kolachev B.A. Hydrogen in metals and alloys. Metal Science and Heat Treatment, 1999, vol. 41, nos. 3-4, pp. 93-100. DOI: 10.1007/BF02467692.

Smirnov S.V. et al. / Energy of activation of titanic alloys of VT5 and VT1-0 at the short-term creep in air and argon. 
11. Reis D.A.P., Silva C.R.M., Nono M.C.A., Barboza M.J.R., Neto F., Perez E.A.C. Effect of environment on the creep behavior of the Ti-6Al-4V alloy. Materials Science and Engineering A-Structural Materials Properties Microstructure and Processing, 2005, vol. 399, nos. 1-2, pp. 276-280. DOI: 10.1016/j.msea.2005.03.073.

12. Lokoshchenko A.M., Il'In A.A., Mamonov A.M., Nazarov V.V. Analysis of the creep and long-term strength of VT6 titanium alloy with preliminarily injected hydrogen. Materials Science, 2008, vol. 44, no. 5, pp. 700-707. DOI: 10.1007/s11003-009-9128-0.

13. Paton N.E., Williams J.C. Hydrogen in Metals, eds. I.M. Bernstein and A.W. Thompson, Metals Park, OH, ASM, 1974, pp. 409-431.

14. Wasz M.L., Brotzen F.R., McLellan R.B., Griffin A.J.Jr. Effect of oxygen and hydrogen on mechanical properties of commercial purity titanium. Int. Mater. Rev., 1996, vol. 41, no. 1, pp. $1-12$.

15. Beevers C.J., Warren M.R., Edmonds D.V. Fracture of titanium-hydrogen alloys. Journal of the Less-Common Metals, 1968, vol. 14, no. 4, pp. 387-396. DOI: 10.1016/0022-5088(68)90162-8.

16. Makarov A.V., Gorkunov E.S, Kogan L.Kh. Application of the eddy-current method for estimating the wear resistance of hydrogen-alloyed beta-titanium alloy BT35. Russian Journal of Nondestructive Testing, 2007, vol. 43, no. 1, pp. 21-26. DOI: 10.1134/S1061830907010032.

17. Suzuki H., Fukushima H., Takai K. Role of Hydrides and Solute Hydrogen in Embrittlement of Pure Titanium. Journal of the Japan Institute of Metals and Materials, 2015, vol. 79, no. 3, pp. 82-88. DOI: 10.2320/jinstmet.JC201402.

18. Smirnov S.V., Zamaraev L.M., Matafonov P.P. Short-term creep of a VT1-0 titanium alloy during heating in a hydrogen atmosphere. Russian Metallurgy (Metally), 2010, vol. 2010, no. 1, pp. 67-70. DOI: 10.1134/S0036029510010131.

19. Reis D.A.P., Neto C.M., Nono M.D.C.A., Barboza M.J.R., Da Silva C.R.M., Neto F.P. Development of a system to creep tests in controlled atmosphere. In: Proceedings of the 65th ABM International Congress, 18th IFHTSE Congress and 1st TMS/ABM International Materials Congress, Rio de Janeiro, Brazil, 26-30 July 2010, vol. 5, pp. 4229-4235.

20. Evans W.J., Jones J.P., Williams S. The interactions between fatigue, creep and environmental damage in Ti 6246 and Udimet 720Li. International Journal of Fatigue, 2005, vol. 27, iss. 10-12, pp. 1473-1484. DOI: 10.1016/j.ijfatigue.2005.06.029.

21. Smirnov S.V., Zamaraev L.M., Matafonov P.P. Short-term thermal cyclic creep and fracture of a VT1-0 titanium alloy in a hydrogen atmosphere. Russian Metallurgy (Metally), 2012, vol. 2012, no. 3, pp. 255-257. DOI: 10.1134/S0036029512030123.

22. Sherby O.D., Lytton J.L, Dorn J.E. Activation energies for creep of high-purity aluminum. Acta Metallurgica, 1957, vol. 5, no. 4, pp. 219-227. DOI: 10.1016/0001-6160(57)90169-4.

23. Rozenberg V.M. Osnovy zharoprochnosti metallicheskikh splavov [Basics of HighTemperature Strength of Metal Alloys]. M., Metallurgiya Publ., 1973, 325 p. (In Russian).

24. $\quad$ Frost H.J., Ashby M.F. Deformation-Mechanism Maps, Pergamon Press, Oxford, 1982.

25. McLean D. Mechanical Properties of Metals, John Wiley \& Sons Inc., New York and London, 1962, $403 \mathrm{p}$.

26. Doner M., Conrad H. Deformation Mechanisms in Commercial Ti-50A (0.5 at. pct $\left.\mathrm{O}_{\text {eq }}\right)$ at Intermediate and High Temperatures (0.3-0.6 $\left.\mathrm{T}_{\mathrm{m}}\right)$. Metallurgical Transactions, 1973, vol. 4, iss. 12, pp. 2809-2817. DOI: 10.1007/BF02644581.

27. Goswam T. Damage Development under Creep-Fatigue in a Titanium and a Superalloy. High Temperature Materials and Processes, 1995, vol. 14, no. 2, pp. 47-56. DOI: 10.1515/HTMP.1995.14.2.47.

28. Sanchez J.N., De Fontaine D., Anomalous diffusion in omega forming systems. Acta Metallurgia, 1978, vol. 26, no. 7, pp. 1083-1095. 
Подана в журнал: 11.11 .2016

УДК 620.172.251:669.295

DOI: $10.17804 / 2410-9908.2016 .6 .100-110$

\title{
ЭНЕРГИЯ АКТИВАЦИИ ТИТАНОВЫХ СПЛАВОВ ВТ1-0 И ВТ5 ПРИ КРАТКОВРЕМЕННОЙ ПОЛЗУЧЕСТИ В ВОЗДУХЕ И АРГОНЕ
}

\author{
С. В. Смирнов ${ }^{1}$, Л. М. Замараев ${ }^{1}$ \\ Федеральное государственное бюджетное учреждение науки \\ Институт машиноведения Уральского отделения Российской академии наук, \\ ул. Комсомольская, 34, Екатеринбург, Российская Федерация \\ *Ответственный автор. Электронная почта: svs@imach.uran.ru; \\ адрес для переписки: ул. Комсомольская, 34, 620049, Екатеринбург, Российская Федерация. \\ Тел.: +7 (343) 375-35-96; факс: +7 (343) 374-53-30
}

Представлены результаты испытаний кратковременной ползучести технически чистого титана ВТ1-0 и сплава ВТ5 диапазоне температур от 673 до 1323 К при номинальных растягивающих напряжениях от 4,45 до 9,36 МПа в воздухе и аргоне. Результаты экспериментов показали значительное снижение скорости ползучести при нагружении в среде аргона по сравнению с испытаниями на воздухе. Значения энергии активации, определенной в испытаниях со ступенчатым изменением температуры по методу Дорна, для исследованных материалов в воздухе и аргоне имеют близкие значения 250-300 кДж/моль, что свидетельствует об одинаковом механизме ползучести.

Ключевые слова: титановые сплавы, влияние окружающей среды на ползучесть, энергия активачии ползучести.

\section{1. Введение}

В связи с расширением области применения титановых сплавов интерес к их поведению при ползучести не ослабевает и в настоящее время. Об этом свидетельствует ряд публикаций, описывающих результаты исследований в широком диапазоне температур, начиная с комнатных до высоких при статическом и циклическом характере нагружения [1-6]. Важной особенностью титана и его сплавов является способность к активному газопоглощению при повышении температуры нагрева: водорода - при температуре свыше 323-343 К, кислорода - при 673-773 К, азота - при 873-973 К. Поэтому защита от взаимодействия с активными газами, содержащимися в воздухе, практически требуется уже при температурах 673 К и выше [7, 8]. Наибольшее количество исследований посвящено ползучести титановых сплавов в перечисленных выше активных средах, которые, как правило, снижают скорость ползучести [4, 9-12] за счет образования с титаном твердых растворов внедрения и высокопрочных частиц оксидов, гидридов и нитридов, которые после охлаждения могут существенным образом изменять физико-механические свойства сплавов [13-17]. Следует отметить, что зависимость скорости кратковременной ползучести титана в водороде имеет температурный диапазон, зависящий от приложенного напряжения, где она аномально повышается [18] по аналогии с известным эффектом «водородного пластфицирования» [5].

Сведения о влиянии нейтральных газовых сред на ползучесть титана и его сплавов в научно-технических публикациях крайне немногочисленны. Из известных следует отметить исследования авторов работ $[6,19]$, которые установили, что долговечность при ползучести $(\alpha+\beta)$ сплава Ti-6Al-4V в аргоне выше по сравнению с воздухом из-за отсутствия окисления поверхности образцов. Снижение циклической долговечности в вакууме по сравнению с воз- 
opent-access journal

духом, обнаруженное в [20], также объяснялось ускорением накопления поврежденности в окислительной среде.

Цель данной статьи - изучить влияние аргона на величину энергии активации при кратковременной высокотемпературной ползучести технически чистого титана ВТ1-0 и сплава ВТ5, относящихся к однофазным $\alpha$-сплавам, в сопоставлении с результатами исследований ползучести в воздушной среде.

\section{2. Материал и методики}

Образцы для исследований были изготовлены из горячепрессованных прутков диаметром 12 мм технически чистого титана ВТ1-0 и сплава ВТ5. Химический состав материалов по марке изготовителя ВТ1-0: $\mathrm{Al}-0,028 ; \mathrm{Si}-0,002 ; \mathrm{Fe}-0,036 ; \mathrm{C}-0,008 ; \mathrm{O}_{2}-0,115$; $\mathrm{H}_{2}-0,003 ; \mathrm{Cr}+\mathrm{Mn}-0,012 ; \mathrm{Cu}+\mathrm{Ni}-0,015 \% ; \mathrm{Ti}$ - остальное. Сплав $\mathrm{BT} 5: \mathrm{Al}-5,563 ; \mathrm{Si}-0,145$; $\mathrm{Fe}-0,3 ; \mathrm{C}-0,09 ; \mathrm{O}_{2}-0,18 ; \mathrm{H}_{2}-0,012 ; \mathrm{Cr}-0,28 ; \mathrm{Ni}-0,045 \% ; \mathrm{Ti}$ - остальное. Температура полиморфного $\alpha \rightarrow \beta$ превращения при нагреве титана ВТ1-0, при которой тип кристаллической решетки меняется с гексагональной плотноупакованной на кубическую объёмноцентрированную, составляет 1160 - 1170 К. Также как и технически чистый титан, сплав ВТ5 является однофазным, полиморфное $\alpha \rightarrow \beta$ превращение в нем происходит при более высокой температуре 1250-1300 К, что обусловлено содержанием в сплаве алюминия.

Образцы для испытаний на ползучесть имели размеры рабочей части: длина 50 мм, диаметр 5 мм. Для крепления образцов в захватах испытательной установки они имели на концах утолщения диаметром 8 мм с нарезанной резьбой.

Испытания проводились на специализированном стенде (рис. 1), позволяющем непрерывно отслеживать удлинение образцов в процессе их нагрева в средах различных газов при фиксированных номинальных растягивающих напряжениях [21].

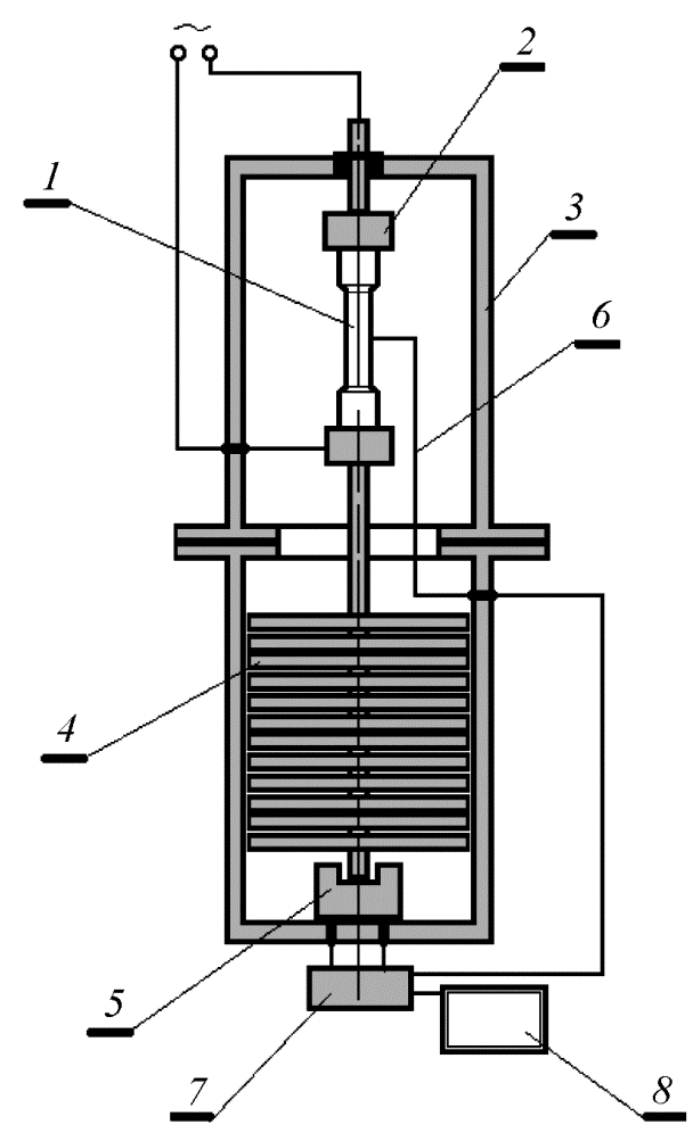

Рис. 1. Схема стенда

Smirnov S.V. et al. / Energy of activation of titanic alloys of VT5 and VT1-0 at the short-term creep in air and argon. 
Для проведения испытаний образец (1) устанавливается в устройстве для подвески образца (2) и закрывается колпаком, образующим герметичную камеру (3). Растягивающее усилие создается набором свинцовых грузов (4). Образец нагревается пропусканием переменного тока через силовой трансформатор с автоматизированным регулированием силы тока до 750 А. Температура нагрева контролируется хромель-алюмелевой термопарой (б), привариваемой к каждому образцу в середине его рабочей части. Визуальное наблюдение за процессом испытаний осуществляется через кварцевые окна в колпаке. Изменение удлинения образца фиксируется с помощью резистивного датчика (5). Датчик и термопара подключены к компьютеру (8) через преобразователь сигнала (7). Воздух из рабочей камеры вытесняется газообразным аргоном из баллона с избыточным давлением 0,5 МПа, которое затем поддерживается постоянным в процессе испытания.

Для определения скорости ползучести использовали метод Дорна [22], при котором в процессе нагрева образцов при постоянных номинальных растягивающих напряжениях температуру изменяли скачкообразно. Скачок температуры $\Delta T$ составлял 30 К и достигался фиксированным изменением силы тока. За периодом скачкообразного увеличения температуры следовал период выдержки. Суммарное время нагрева и выдержки для каждого этапа составляло 500 с. Поскольку скачок температур незначителен, а напряжение до и после изменения температуры оставалось постоянным, изменением модуля упругости и субструктуры можно пренебречь. При таком допущении изменение скорости ползучести определяется только изменением температуры. Испытания образцов проводили в диапазоне температур от 673 до 1323 К при номинальных растягивающих напряжениях $\sigma=4,45 ; 6,91$ и 9,36 МПа, которые были ниже предела текучести исследуемых сплавов при максимальных температурах испытаний $\left(\sigma_{m}=12\right.$ МПа для ВТ1-0 и 45 МПа для ВТ5).

В процессе исследований с целью получения данных для статистической обработки при каждом режиме проводилось по 3 эксперимента. На рис. 2 в качестве примера исходных экспериментальных данных приведены изменения показаний датчика удлинения (1) в процессе нагрева образца из сплава ВТ5 при номинальном растягивающем напряжении 9,36 МПа.

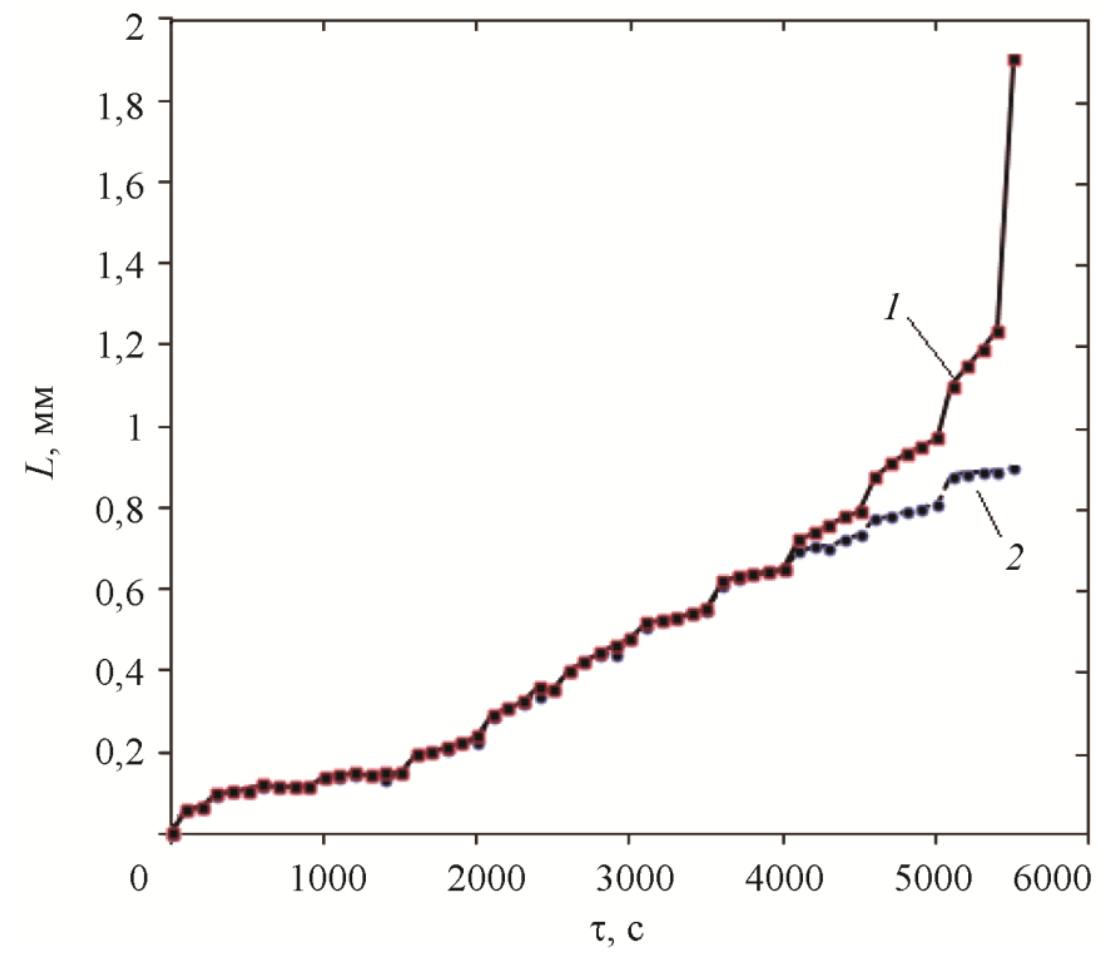

Рис. 2. Диаграммы показаний датчика удлинения при нагреве образца из сплава Ti-5Al в среде аргона при действии номинального растягивающего напряжения 9,36 МПа (1) и без нагрузки (2)

Smirnov S.V. et al. / Energy of activation of titanic alloys of VT5 and VT1-0 at the short-term creep in air and argon. 
Для того чтобы исключить вклад величины температурной деформации образца и элементов оснастки в фиксируемое удлинение каждый эксперимент с нагруженным образцом дублировали экспериментом без подвешенных грузов при тех же временных и температурных параметрах (2). Разницу в измерениях относили на счет удлинения образца при ползучести.

Испытания проводили только на стадии равномерного удлинения рабочей части образца, не достигая стадии локализации деформации и образования шейки. Схема на рис. 3 поясняет методику обработки экспериментальных данных на одной из ступеней нагрева.

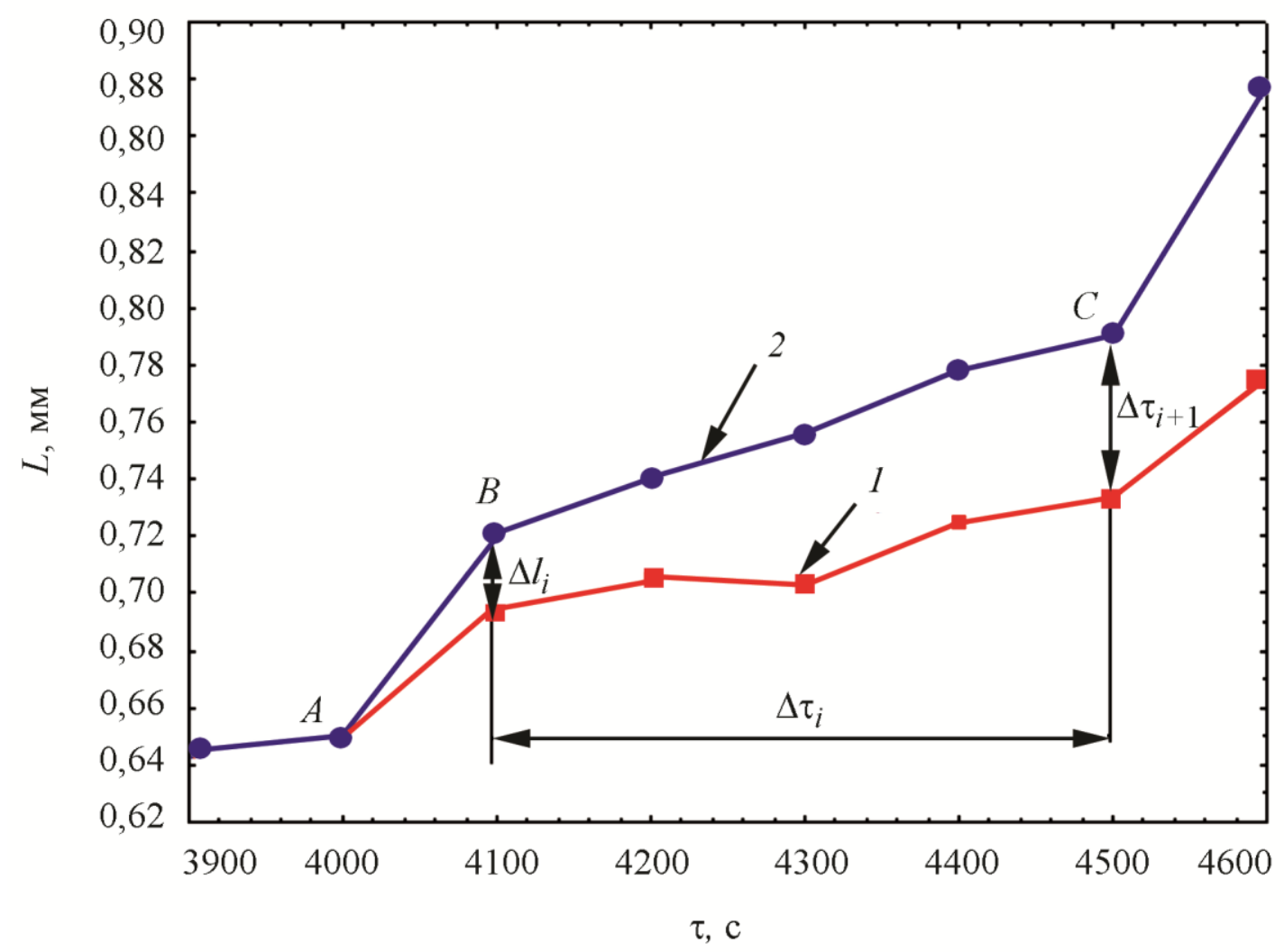

Рис.3. К методике обработки экспериментальных данных. Участок диаграммы показаний датчика удлинения при нагреве образца из сплава ВТ5 в среде аргона при действии номинального растягивающего напряжения 9,36 МПа (1) и без нагрузки (2)

Для приведенного случая на 4000 секунде нагрева $(A)$ была увеличена сила тока, что вызвало скачкообразный нагрев образца с 1073 до 1103 К $(B)$, после чего в интервале времени $\Delta \tau=4100-4500$ с делали выдержку при постоянной температуре 1103 К.

На диаграмме выделяли прямолинейный участок $B C$, на котором рассчитывали относительное удлинение образца $\Delta \varepsilon_{i}$ за счет ползучести на 1-м этапе испытания:

$$
\Delta \varepsilon_{i}=\frac{\Delta l_{i+1}-\Delta l_{i}}{L_{i}}
$$

где $\Delta l_{i+1}$ и $\Delta l_{i}$, - удлинение образца за счет ползучести в конце и начале выдержки при постоянной температуре $T_{i} ; L_{i}-$ длина рабочей части образца в начале каждого периода выдержки.

Среднюю скорость ползучести на участке выдержки рассчитывали по формуле:

$$
v_{i}=\frac{\Delta \varepsilon_{i}}{\Delta \tau_{i}}, 1 / \mathrm{c},
$$

Smirnov S.V. et al. / Energy of activation of titanic alloys of VT5 and VT1-0 at the short-term creep in air and argon. 
где $\Delta \tau_{i}-$ время паузы, с; $\Delta \varepsilon_{i}-$ относительное удлинение образца за счет ползучести на $i$-й ступени изменения температуры.

Энергию активации $\Delta H_{i}$ установившейся ползучести на $i$-й ступени нагрева рассчитывали по формуле [22, 24]:

$$
\Delta H_{i}=\frac{R \ln \left(\frac{v_{i}}{v_{i+1}}\right)}{\Delta \tau_{i}}, \text { кДж/моль, }
$$

где $R=0,0083144598$ кДж/(моль $\cdot \mathrm{K})-$ универсальная газовая постоянная.

Для каждого сплава и среды испытаний значения энергии активации, полученные по формуле (3), усреднялись сначала за весь период ползучести, а затем и по всем исследованным номинальным напряжениям.

\section{3. Результаты исследований и обсуждение}

Результаты экспериментов по определению скорости ползучести сплавов ВТ5 и ВТ1-0 в исследованных интервалах температур приведены в виде точек на рис. 4.

Основываясь на полученных данных, определяем зависимость скорости ползучести $(v)$ от энергии активации $(\Delta H)$, номинальных растягивающих напряжений $(\sigma)$ и температуры нагрева $(T)$. Для ее описания выбрана известная $[23,25]$ экспоненциальная зависимость:

$$
v=A \sigma^{n} \exp \left(-\frac{\Delta H}{R T}\right), 1 / \mathrm{c},
$$

где $A, n$ - эмпирические коэффициенты аппроксимации.

Для определения коэффициентов этой зависимости, полученные экспериментальные данные были подвергнуты регрессионному анализу в программном комплексе Microsoft Excel. С целью повышения точности определения эмпирических коэффициентов $A, n$, их определяли из линеризованной зависимости:

$$
\ln v=\ln A+n \ln \sigma-\frac{\Delta H}{R T} .
$$

Значения этих коэффициентов, усредненные значения энергии активации, а так же температурные пределы в которых они были получены, приведены в таблице.

Значения энергии активации и эмпирических коэффициентов в формуле (4)

\begin{tabular}{|c|l|c|c|c|c|}
\hline \multirow{2}{*}{ Сплав } & \multicolumn{1}{|c|}{ Среда } & $\begin{array}{c}\Delta H_{\mathrm{cp}}, \\
\text { кДж/моль }\end{array}$ & $A \times 10^{-4}$ & $n$ & $\begin{array}{c}\text { Пределы } \\
\text { температур, К }\end{array}$ \\
\hline \multirow{2}{*}{ ВТ1-0 } & Воздух & 273 & 8,7 & 6,77 & $850-1050$ \\
\cline { 2 - 6 } & Аргон & 302 & 13,5 & 2,83 & $950-1150$ \\
\hline \multirow{2}{*}{ ВT5 } & Воздух & 260 & 8,06 & 4,17 & $900-1100$ \\
\cline { 2 - 6 } & Аргон & 307 & 9,48 & 3,09 & $1000-1200$ \\
\hline
\end{tabular}

Smirnov S.V. et al. / Energy of activation of titanic alloys of VT5 and VT1-0 at the short-term creep in air and argon. 

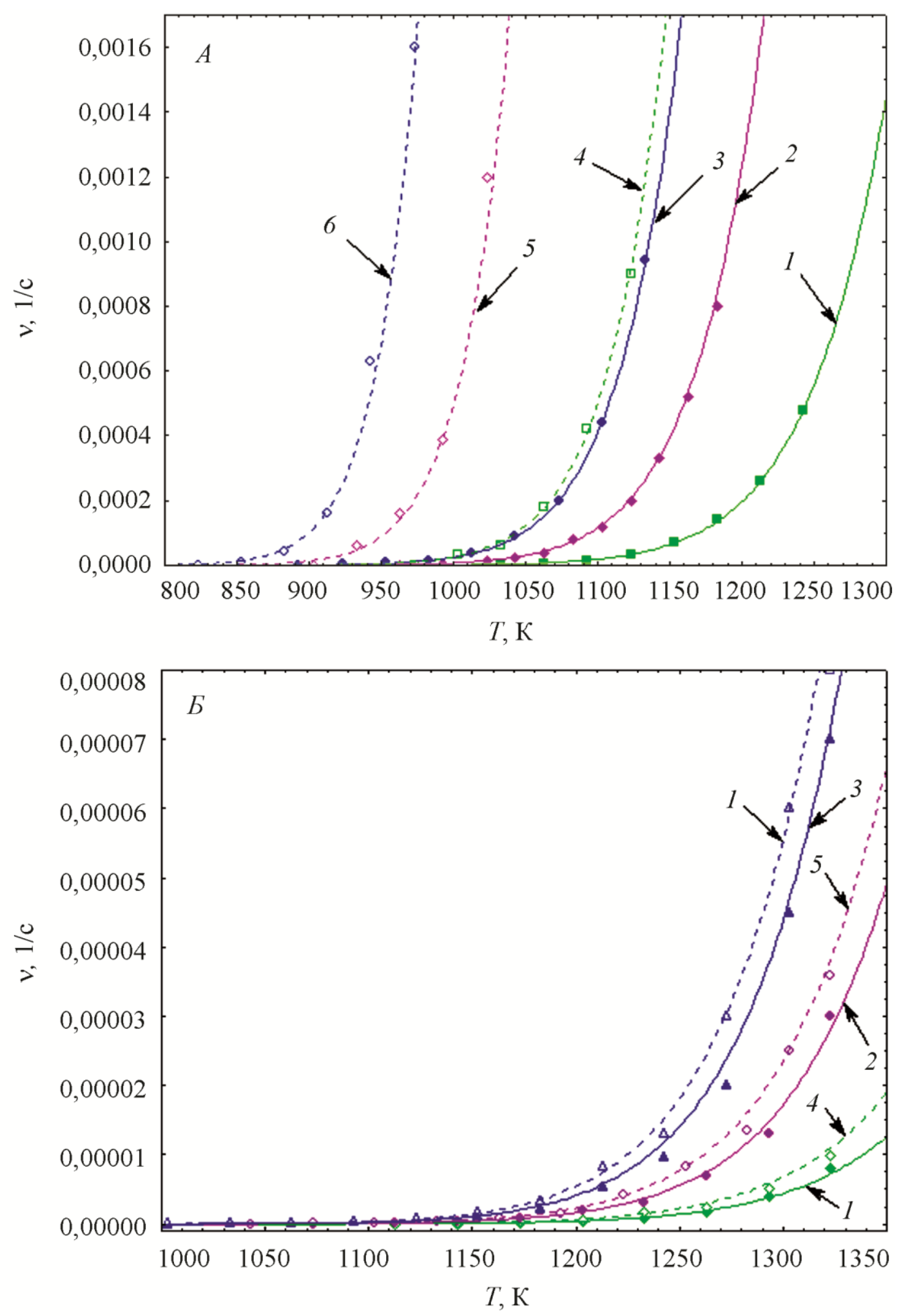

Рис. 4. Экспериментальные данные и расчетные зависимости скорости деформации титановых сплавов ВТ5 и ВТ1-0 на установившейся стадии ползучести от температуры нагрева при различных растягивающих напряжениях:

A. При нагреве в воздушной среде: $1-$ ВТ5 $\sigma=4,45$ МПа; $2-$ ВТ5 $\sigma=6,91$ МПа; 3 - ВТ5 $\sigma=$ 9,36 МПа; 4 - ВТ1-0 $\sigma=$ 4,45 МПа; 5 - ВТ1-0 $\sigma=6,91$ МПа; 6 - ВТ1-0 $\sigma=9,36$ МПа;

Б. При нагреве в среде аргона: 1 - ВТ5 $\sigma=4,45$ МПа; 2 - ВТ5 $\sigma=6,91$ МПа; 3 - ВТ5 $\sigma=$ 9,36 МПа; 4 - ВТ1-0 $\sigma=$ 4,45 МПа; 5 - ВТ1-0 $\sigma=6,91$ МПа; 6 - ВТ1-0 $\sigma=$ 9,36 МПа.

Точки - усредненные экспериментальные данные

Smirnov S.V. et al. / Energy of activation of titanic alloys of VT5 and VT1-0 at the short-term creep in air and argon. 
Оценку адекватности полученной аппроксимации осуществляли путем сравнения результатов расчетов по формуле (4) при найденных значениях коэффициентов $A$ и $n$ с данными, полученными по результатам экспериментов при номинальном напряжении 6,91 МПа. При расчетах использовали усредненные значения энергии активации $\Delta H_{\mathrm{cp}}$, приведенные в таблице. В результате было установлено, что указанные коэффициенты аппроксимации обеспечивают точность описания экспериментальных данных с коэффициентом достоверности аппроксимации $R^{2}>0,96$ в исследованных температурных интервалах. На рис. 4 приведены графики зависимостей скоростей ползучести исследованных материалов от температуры испытания, которые были рассчитаны по формуле (4) и данные, полученные по результатам обработки экспериментов.

Как видно из представленных выше результатов, состав газовой среды, в которой проводятся испытания, оказывает существенное влияние на характеристики ползучести. При нагреве образцов из обоих сплавов в среде аргона наблюдалось значительное снижение скорости ползучести $v$ по сравнению с аналогичным нагревом в воздушной среде. При качественной аналогии указанных закономерностей ползучесть в сплаве ВТ5 по сравнению с технически чистым титаном ВТ1-0 фиксируется при более высоких температурах и имеет более низкую скорость, что связано с наличием твердого раствора алюминия в титане.

Для определения механизма ползучести технически чистого титана ВТ1-0 в соответствии с рекомендациями [24] были рассчитаны значения комплекса $\sigma_{S} / \mu\left(\sigma_{S}=\sigma / \sqrt{3}-\right.$ напряжение сдвига; $\mu$ - модуль сдвига при значениях гомологической температуры испытаний $T / T_{m}$ от 0,43 до 0,54 , соответствующие условиям экспериментов ( $T_{m}=1957 \mathrm{~K}$ - температура плавления титана). Значение $\mu$ при температуре испытания определяли по формуле (7) [24]:

$$
\mu=\mu_{0}\left[1+k_{T} \frac{(T-300)}{T_{m}}\right],
$$

где $\mu_{0}=4,36 \cdot 10^{4} \mathrm{MH} / \mathrm{M}^{2}$ - модуль сдвига при $T=300 \mathrm{~K} ; k_{T}=-1,2-$ коэффициент температурной зависимости модуля $\mu$ [27].

Диапазон рассчитанных значений для условий экспериментов составил: $\mu=2,13 \times 10^{4}-$ $2,75 \times 10^{-4} \mathrm{MH} / \mathrm{M}^{2}$ и $\sigma_{S} / \mu=1,8 \times 10^{-4}-3,7 \times 10^{-4}$ (для ВТ1-0).

Это позволило по карте механизмов деформации для титана [24] установить, что в соответствии с классификацией Г.Дж. Фроста и М.Ф. Эшби [24] условия испытаний соответствуют области высокотемпературной ползучести, контролируемой объемной диффузией переползания дислокаций, где справедлива степенная зависимость скорости деформации от приложенного напряжения. Следует также отметить относительную близость значений постоянной Дорна $A$ и энергии активации $\Delta H$ (таблица) с результатами исследований $[24,25]$ $\left(A=7,7 \times 10^{4} ; \Delta H=242\right.$ кДж/моль), выполненными на образцах из более чистого по примесям титана $(99,98 \%)$.

Рассчитанные значения энергии активации $\Delta H$ в диапазоне 260-307 кДж/моль для обоих исследованных материалов в воздухе и аргоне выше значений $\Delta H$, приведенных [24] для механизмов объемной диффузии $(\Delta H=150$ кДж/моль) и для диффузии по границам зерен и дислокационным трубкам ( $\Delta H=97$ кДж/моль). То есть испытания в аргоне не меняют по сравнению с воздухом механизм деформации, хотя при этом значительно снижается скорость ползучести. Последнее можно объяснить из общих представлений об определяющем влиянии состояния поверхности на процессы деформации металлических материалов, в данном случае - процессов окисления $[22,26]$. 


\section{4. Заключение}

Энергия активации высокотемпературной кратковременной ползучести для технически чистого титана ВТ1-0 и сплава ВТ5 в воздухе и аргоне имеет близкие значения в интервале 250-300 кДж/моль, что соответствует области высокотемпературной ползучести, контролируемой объемной диффузией переползания дислокаций, где справедлива степенная зависимость скорости деформации от приложенного напряжения.

Определены эмпирические коэффициенты в степенной зависимости, описывающие скорость кратковременной ползучести на установившейся стадии в аргоне и воздухе от температуры нагрева и номинального напряжения растяжения. При испытаниях в среде аргона, по сравнению с испытаниями на воздухе, наблюдается снижение скорости ползучести при одинаковых номинальных напряжениях во всем температурном диапазоне испытаний.

\section{Литература}

1. Kumar J., Raman S. G. S., Kumar V. Creep-Fatigue Interactions in Ti-6Al-4V Alloy at Ambient Temperature // Transactions of the Indian Institute of Metals. - 2016. - Vol. 69, no. 2. P. 349-352. - DOI: 10.1007/s12666-015-0766-7.

2. Grabovetskaya G. P., Zabudchenko O. V., Stepanova E. N. Effect of hydrogen on the lowtemperature creep of a submicrocrystalline Ti-6Al-4V alloy // Russian Metallurgy (Metally). 2010. - Vol. 2010, no. 3. - P. 229-234. - DOI: 10.1134/S0036029510030134.

3. High temperature creep and fatigue behavior and life prediction method of a TiAl alloy / H. Yu, C. Dong, Z. Jiao, F. Kong, Y. Chen, Y. Su // Acta Metallurgica Sinica. - 2013. - Vol. 49, no. 11. - P. 1311-1317. - DOI: 10.3724/SP.J.1037.2013.00434.

4. Rosen A., Rottem A. The effect of high temperature exposure on the creep resistance of Ti6Al4V alloy // Mater. Sci. Eng. A. - 1976. - Vol. 22, no. C. - P. 23-294. - DOI: 10.1016/00255416(76)90132-4.

5. Influence of hydrogen on the plasticity and elastic stiffness of technical titanium VT1-0 at temperatures up to $750^{\circ} \mathrm{C} / \mathrm{Yu}$. A. Aksenov, I. O. Bashkin, V. L. Kolmogorov, Ye. G. Ponyatovskiy, G. G. Taluts, V. K. Kataya, I. V. Levin, Yu. I. Potapenko, A. N. Trubin // Physics of Metals and Metallography. - 1989. - Vol. 67, no. 5. - P. 157-163.

6. The oxidation effect in the titanium alloy at high temperature / D. A. P. Reis, C. de Moura Neto, F. P. Neto., M. J. R. Barboza, C. R. M. da Silva // SAE Technical Papers. 2007.

7. Колычев Б. А. Водородная хрупкость металлов. - М. : Металлургия, 1985. - 286 с.

8. Механические свойства легких сплавов при температурах и скоростях обработки давлением : справочник / под. ред. П. Г. Микляева. - М. : Металлургия, 1994. - 280 с.

9. On the mechanisms of superplasticity in Ti-6Al-4V / E. Alabort, P. Kontis, D. Barba, K. Dragnevski, R. C. Reed // Acta Materialia. - 2016. - Vol. 105, no. 1. - P. 449-463.

10. Kolachev B. A. Hydrogen in metals and alloys // Metal Science and Heat Treatment. 1999. - Vol. 41, nos. 3-4. - P. 93-100. - DOI: 10.1007/BF02467692.

11. Effect of environment on the creep behavior of the Ti-6Al-4V alloy / D. A. P. Reis, C. R. M. Silva, M. C. A. Nono, M. J. R. Barboza, F. Neto, E. A. C. Perez // Materials Science and Engineering A-Structural Materials Properties Microstructure and Processing. - 2005. - Vol. 399, no. 1-2. - P. 276-280. - DOI: 10.1016/j.msea.2005.03.073.

12. Analysis of the creep and long-term strength of VT6 titanium alloy with preliminarily injected hydrogen / A. M. Lokoshchenko, A. A. Il'In, A. M. Mamonov, V. V. Nazarov // Materials Science. - 2008. - Vol. 44, no. 5. - P. 700-707. - DOI: 10.1007/s11003-009-9128-0.

13. Paton N. E., Williams J. C. Hydrogen in Metals / ed. by I. M. Bernstein, A. W. Thompson. Metals Park, OH, ASM, 1974. - P. 409-431. 
14. Effect of oxygen and hydrogen on mechanical properties of commercial purity titanium / M. L. Wasz, F. R. Brotzen, R. B. McLellan, A. J. Griffin Jr // Int. Mater. Rev. - 1996. - Vol. 41, no. 1. - P. 1-12.

15. Beevers C. J., Warren M. R., Edmonds D. V. Fracture of titanium-hydrogen alloys // Journal of the Less-Common Metals. - 1968. - Vol. 14, no. 4. - P. 387-396. - DOI: 10.1016/00225088(68)90162-8.

16. Makarov A. V., Gorkunov E. S, Kogan L. Kh. Application of the eddy-current method for estimating the wear resistance of hydrogen-alloyed beta-titanium alloy BT35 // Russian Journal of Nondestructive Testing. - 2007. - Vol. 43, no. 1. - P. 21-26. DOI: 10.1134/S1061830907010032.

17. Suzuki H., Fukushima H., Takai K. Role of hydrides and solute hydrogen in embrittlement of pure titanium // Journal of the Japan Institute of Metals. - 2015. - Vol. 79, no. 3. - P. 82-88. DOI: 10.2320/jinstmet.JC201402.

18. Smirnov S. V., Zamaraev L. M., Matafonov P. P. Short-term creep of a VT1-0 titanium alloy during heating in a hydrogen atmosphere // Russian Metallurgy (Metally). - 2010. - Vol. 2010, no. 1. - P. 67-70. - DOI: 10.1134/S0036029510010131.

19. Development of a system to creep tests in controlled atmosphere / D. A. P. Reis, C. M. Neto, M. D. C. A. Nono, M. J. R. Barboza, C. R. M. da Silva, F. P. Neto // 65th ABM International Congress, 18th IFHTSE Congress and 1st TMS/ABM International Materials Congress, Rio de Janeiro, Brazil, 26-30 July, 2010 : proceedings. - 2010. - Vol. 5. - P. 4229-4235.

20. Evans W. J., Jones J. P., Williams S. The interactions between fatigue, creep and environmental damage in $\mathrm{Ti} 6246$ and Udimet 720Li // International Journal of Fatigue. - 2005. Vol. 27. - P. 1473-1484. - DOI: 10.1016/j.ijfatigue.2005.06.029.

21. Smirnov S. V., Zamaraev L. M., Matafonov P. P. Short-term thermal cyclic creep and fracture of a VT1-0 titanium alloy in a hydrogen atmosphere // Russian Metallurgy (Metally). - 2012. Vol. 2012, no. 3. - P. 255-257. - DOI: 10.1134/S0036029512030123.

22. Sherby O. D., Lytton J. L, Dorn J. E. Activation energies for creep of high-purity aluminum // Acta Metallurgica. - 1957. - Vol. 5, no. 4. - P. 219-227. - DOI: 10.1016/0001-6160(57)90169-4.

23. Розенберг В. М. Основы жаропрочности металлических сплавов. - М. : Металлургия, 1973. $-325 \mathrm{c}$.

24. Frost H. J., Ashby M. F. Deformation-Mechanism Maps. - Oxford : Pergamon Press, 1982.

25. McLean D. Mechanical Properties of Metals. - New York, London : John Willey\&Sons Inc, 1962. $-403 \mathrm{p}$.

26. Doner M., Conrad H. Deformation Mechanisms in Commercial Ti-50A (0.5 at. pct $\left.\mathrm{O}_{\mathrm{eq}}\right)$ at Intermediate and High Temperatures (0.3-0.6 $\left.\mathrm{T}_{\mathrm{m}}\right)$ // Metallurgical Transactions. - 1973. - Vol. 4, iss. 12. - P. 2809-2817. - DOI: 10.1007/BF02644581.

27. Goswam T. Damage Development under Creep-Fatigue in a Titanium and a Superalloy // High Temperature Materials and Processes. - 1995. - Vol. 14, no. 2. - P. 47-56. DOI: 10.1515/HTMP.1995.14.2.47.

28. Sanchez J. N., De Fontaine D. Anomalous diffusion in omega forming systems // Acta Metallurgia. - 1978. - Vol. 26, iss. 7. - P. 1083-1095. 